\title{
Article
}

\section{Synthesis of Zinc Phosphonated Poly(ethylene imine) and Its Fire- Retardant Effect in Low-Density Polyethylene}

\author{
Zhang, Y, Liu, X, Cao, Z, Fang, Z, Hull, TR and Stec, AA
}

Available at https://clok.uclan.ac.uk/16624/

Zhang, Y, Liu, X, Cao, Z, Fang, Z, Hull, TR orcid iconORCID: 0000-0002-79704208 and Stec, AA orcid iconORCID: 0000-0002-6861-0468 (2015) Synthesis of Zinc Phosphonated Poly(ethylene imine) and Its Fire-Retardant Effect in Low-Density Polyethylene. Industrial \& Engineering Chemistry Research, 54 (13). pp. 3247-3256. ISSN 0888-5885

It is advisable to refer to the publisher's version if you intend to cite from the work. http://dx.doi.org/10.1021/ie504200y

For more information about UCLan's research in this area go to http://www.uclan.ac.uk/researchgroups/ and search for <name of research Group>.

For information about Research generally at UCLan please go to http://www.uclan.ac.uk/research/

All outputs in CLoK are protected by Intellectual Property Rights law, including Copyright law. Copyright, IPR and Moral Rights for the works on this site are retained by the individual authors and/or other copyright owners. Terms and conditions for use of this material are defined in the policies page.

\section{CLoK}

Central Lancashire online Knowledge www.clok.uclan.ac.uk

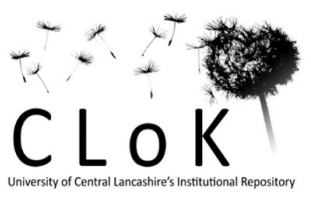




\title{
Synthesis of zinc phosphonated poly(ethylene imine) and its fire
}

\author{
retardant effect in low density polyethylene
}

Yan Zhang ${ }^{1,3}$, Xiaonan $\mathrm{Li}^{1}$, Zhenhu $\mathrm{Cao}^{2}$, Zhengping Fang ${ }^{1,2^{*}}$, T Richard Hull ${ }^{3 *}$, Anna

$$
\text { A. } \text { Stec }^{3}
$$

1 Lab of Polymer Materials and Engineering, Ningbo Institute of Technology, Zhejiang University, Ningbo 315100, China

2 MOE Key Laboratory of Macromolecular Synthesis and Functionalization, Department of

Polymer Science and Engineering, Zhejiang University, Hangzhou 310027, China

3 Centre for Fire and Hazards Science, University of Central Lancashire, Preston PR1 2HE, UK

E-mail: zpfang@zju.edu.cn, trhull@uclan.co.uk

\begin{abstract}
A novel oligomeric intumescent fire retardant chelate, zinc phosphonated poly(ethylene imine) (Zn-PEIP), with a changeable $\mathrm{Zn}^{2+}$ loading, was synthesized. The chemical structure of Zn-PEIP was confirmed by FTIR, ${ }^{13} \mathrm{C}$ NMR and ${ }^{31} \mathrm{P}$ NMR spectroscopy. The thermal behavior and fire retardancy of low density polyethylene (LDPE) containing $25 \mathrm{wt} \% \mathrm{Zn}$-PEIPs with different amounts of $\mathrm{Zn}^{2+}$ were investigated by thermogravimetric analysis (TGA), limiting oxygen index (LOI) and cone calorimetry. The TGA results showed that higher concentrations of $\mathrm{Zn}^{2+}$ improved the thermal stability and increased the residue yield of LDPE. However, the data from the LOI and cone calorimetry showed that there is an optimum concentration of $\mathrm{Zn}^{2+}$ for the best fire retardant performance of LDPE. This is ascribed to the high crosslink density resulting from zinc bridges, preventing normal swelling of the intumescent system. The surface morphology of the char was characterized by digital photographs and scanning electron microscopy (SEM). This confirmed the optimum intumescence, coherent and strong barrier layer formation at an intermediate $\mathrm{Zn}^{2+}$ loading.
\end{abstract}

Keywords: Zinc chelate; Low density polyethylene; Fire retardant; Mannich condensation; Flammability 


\section{Introduction}

Intumescent fire retardants (IFRs) are often phosphorus-nitrogen containing compounds, which are considered as promising candidates to substitute for the halogen-containing flame retardants, since there are no known environmental problems associated with their use ${ }^{1-4}$. The proposed mechanism of the IFR is based on swollen char acting as physical barrier, which slows down heat and mass transfer between the gas and the condensed phases ${ }^{5}$. However, an effective IFR loading is about $30 \mathrm{phr}$ or more, which may be detrimental to the mechanical properties of the plastic, and make it more difficult to recycle. Therefore, attention was focused on how to improve the efficiency of the IFRs and so reduce their loading ${ }^{6-8}$.

Recently, compounds containing metallic elements such as transition metal oxides and metal sulfates have been utilized for improving the flame retardancy of the IFR-polymer system ${ }^{9-11}$. These metal compounds can not only act in the vapor phase, but also in the condensed phase and at the gas/solid interface, so as to reduce the flammability through a chemical and/or physical mechanism ${ }^{12-16}$.

Wu reported that zinc and nickel salts increased the limiting oxygen index (LOI) and decreased the heat release rate (HRR) in blends of polypropylene (PP)/ammonium polyphosphate (APP)/dipentaerythritol (DPER) ${ }^{17}$. Lewin reported that divalent and multivalent metal oxides could enhance the fire retardancy of intumescent systems based on APP and pentaerythritol (PER) in $\mathrm{PP}^{18}$.

Although the metal compounds mentioned above can improve the fire retardancy of the IFR in a polymer, such blending of IFR and metal compounds can result in problems of heterogeneous distribution and incompatibility, reducing the fire retardant effectiveness. To avoid this, an IFR molecule with a metal ion in one molecule, zinc-tetraethyl (1,2-phenylenebis(azanediyl)) bis (2-hydroxylphenylmethylene) diphosphonate (Zn-TEPAPM) (Figure 1), was developed in our earlier work ${ }^{9}$. This is a metal chelate containing phosphorus, nitrogen and a metallic element. However, the fire retardancy of Zn-TEPAPM was not as good as expected ${ }^{9}$. When the content of Zn-TEPAPM was $25 \mathrm{wt} \%$ in low density polyethylene (LDPE), the reduction of peak 
heat release rate (PHRR) of LDPE/Zn-TEPAPM was $41 \%$ and the char layer was not continuous, leaving a lot of holes in the residue from the cone calorimeter test. If the content of Zn-TEPAPM was $1 \mathrm{wt} \%$ and was blended with 19 wt $\%$ APP in LDPE, a more intact and continuous char layer was formed and the reduction of PHRR of LDPE/19APP/1Zn-TEPAPM reached 51\%. The reason for such results is ascribed to the effect of different loadings of zinc ions on the fire retardancy of LDPE. However, due to the limitations of the structure of $\mathrm{Zn}$-TEPAPM, it is difficult to change $\mathrm{Zn}^{2+}$ loading in TEPAPM.

Therefore, a novel oligomeric IFR chelate, zinc phosphonated poly(ethylene imine) (Zn-PEIP), containing phosphorus, nitrogen and zinc was designed and synthesized. As Fig. 1 shows, there are multiple coordination positions available on the branched chains of the oligomeric ligand to be chelated with metal ions. This allows the content of metal ions in the molecule to be varied from zero to a saturation value. By changing the zinc ion loading, the fire retardancy of such an oligomeric IFR chelate can be optimized and used to study the structure-property relationships.

The Mannich condensation was used to synthesize the phosphonated poly(ethylene imine) (PEIP), for use as an IFR ligand. Zinc (Zn) was chosen to be chelated with PEIP for its ability to improve the dehydrogenation during thermal degradation ${ }^{19}$. The chemical structure and thermal properties of Zn-PEIP and PEIP were characterized by infrared analysis (IR), NMR (including ${ }^{13} \mathrm{C}-\mathrm{NMR}$ and ${ }^{31} \mathrm{P}-\mathrm{NMR}$ ) and thermogravimetric analysis (TGA). With the varied content of Zn in Zn-PEIP, the effects of Zn-PEIP in low density polyethylene (LDPE) were studied with respect to thermal stabilization, burning behaviour and flame retardancy, using TGA, LOI, the cone calorimeter (CONE), scanning electron microscope (SEM) and X-ray diffraction (XRD). 


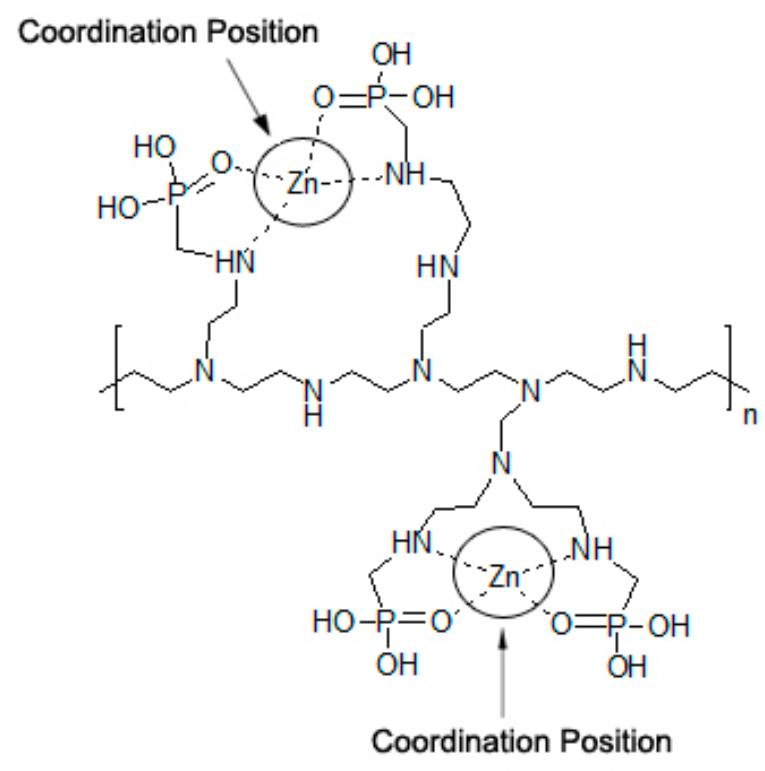

Fig. 1 Proposed structure of Zn-PEIP

\section{Experimental}

\subsection{Materials}

LDPE (2102TN26) with a melt flow index of 2.1-2.9 g/10min and a density of 0.920-0.922 g/m³ was purchased from Sinopec Qilu Company Ltd. (Zibo, China). LDPE grafted with about 1.0 wt\% maleic anhydride (TRD-100L, LDPE-g-MA) was provided by Wujiang Siruida Plastic Industry Co., Ltd. (Wujiang, China). Poly(ethylene imine) (PEI, $M_{\mathrm{w}}=10$ 000, 99 wt\%) was purchased from Aladdin-reagent Co., Ltd. (Shanghai, China). Phosphonic acid (HPO $\left.(\mathrm{OH})_{2}\right)$, concentrated hydrochloric acid solution $(\mathrm{HCl})$, formaldehyde solution $\left(\mathrm{CH}_{2} \mathrm{O}\right)$ of 37 wt\%, diethanolamine and anhydrous ethanol $\left(\mathrm{C}_{2} \mathrm{H}_{5} \mathrm{OH}\right)$ were purchased from Sinopharm Chemical Reagent Co. Ltd. (Shanghai, China). Sodium hydroxide (NaOH) was purchased from Xilong Chemical Reagent Co. Ltd. (Guangzhou, China). Zinc acetate $\left(\mathrm{Zn}\left(\mathrm{OOCCH}_{3}\right)_{2} \cdot 2 \mathrm{H}_{2} \mathrm{O}\right.$, Znac) was purchased from the Yixing 2nd Chemical Reagent Factory (Yixing, China). All chemical reagents were used without further purification. 


\subsection{The synthesis of PEIP and Zn-PEIP}

\subsubsection{The synthesis of PEIP}

PEIP was obtained using the Mannich condensation reaction of poly(ethylene imine) (PEI), formaldehyde and phosphonic acid. $16.4 \mathrm{~g}$ of phosphonic acid and $10 \mathrm{~g}$ of PEI were dissolved in $100 \mathrm{~mL}$ of $\mathrm{HCl}$ in a $250 \mathrm{~mL}$ three-neck flask equipped with a mechanical stirring and reflux condenser. When the above solution was heated to reflux, $32 \mathrm{~mL}$ of $37 \mathrm{wt} \%$ formaldehyde solution was added dropwise into the flask over a period of $1 \mathrm{~h}$. Then, the system was heated under reflux for $1 \mathrm{~h}$. When cooled to room temperature, the mixture was neutralized by diethanolamine and the crude PEIP product precipitated. This was filtered and washed three times using anhydrous ethanol. Finally, the PEIP product was dried under vacuum as a yellow powder with a yield of 95\%. The synthesis route is illustrated in Fig. 2(a).

\subsubsection{The synthesis of Zn-PEIP}

A three-neck flask was charged with different amounts of Znac, 15 g of PEIP, 2 g of $\mathrm{NaOH}$ and $500 \mathrm{~mL}$ of anhydrous ethanol. The mixture was stirred under nitrogen for $7 \mathrm{~h}$ under reflux. After cooling to room temperature, the yellow suspension was filtered and washed thoroughly with anhydrous ethanol, and then dried at $50{ }^{\circ} \mathrm{C}$ under vacuum to a constant weight. In order to prepare the $\mathrm{Zn}$-PEIP with different $\mathrm{Zn}^{2+}$ loadings, the amount of Znac was varied from $0.1 \mathrm{~mol}, 0.07 \mathrm{~mol}, 0.05 \mathrm{~mol}, 0.03 \mathrm{~mol}$, $0.01 \mathrm{~mol}, 0.007 \mathrm{~mol}$ down to $0.003 \mathrm{~mol}$. The corresponding Zn chelates are named as Zn-PEIP-0.1， Zn-PEIP-0.07， Zn-PEIP-0.05， Zn-PEIP-0.03， Zn-PEIP-0.01, Zn-PEIP-0.007 and Zn-PEIP-0.003. The chelation step in the synthesis route is illustrated in Fig. 2(b). 

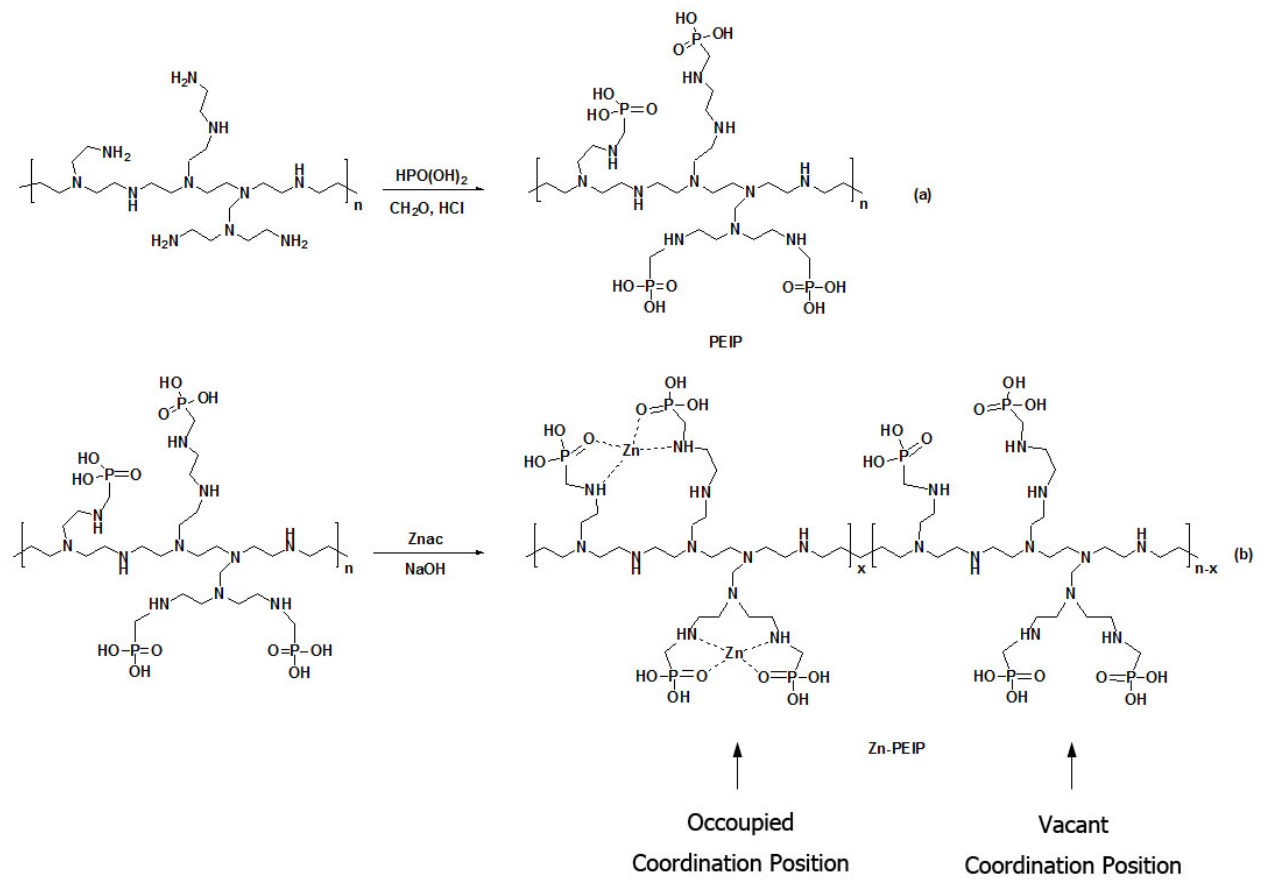

Fig. 2 The synthesis route of PEIP (a) and Zn-PEIP (b)

\subsection{Preparation of fire retardant LDPE composites}

The LDPE composites were prepared via melt compounding at $160{ }^{\circ} \mathrm{C}$ in Thermo-Haake rheomixer with a rotor speed of $60 \mathrm{rpm}$, according to the formulations presented in Table 1. The prepared materials were transferred into a mold and preheated for $5 \mathrm{~min}$ at $120^{\circ} \mathrm{C}$, and then pressed at $15 \mathrm{MPa}$ for $8 \mathrm{~min}$, followed by pressing at room temperature under the same pressure for $5 \mathrm{~min}$.

Table 1 The formulation of flame retardant LDPE with Zn-PEIP and PEIP

\begin{tabular}{lcccc}
\hline \multicolumn{1}{r}{ Sample ID } & LDPE /wt\% & LDPE-g-MA /wt\% & PEIP /wt\% & Zn-PEIP /wt\% \\
\hline LDPE & 95 & 5 & & \\
LDPE/Zn-PEIP-0.1 & 70 & 5 & & 25 \\
LDPE/Zn-PEIP-0.07 & 70 & 5 & & 25 \\
LDPE/Zn-PEIP-0.05 & 70 & 5 & & 25 \\
LDPE/Zn-PEIP-0.03 & 70 & 5 & & 25 \\
LDPE/Zn-PEIP-0.01 & 70 & 5 & & 25 \\
LDPE/Zn-PEIP-0.007 & 70 & 5 & 25 & 25 \\
LDPE/Zn-PEIP-0.003 & 70 & 5 & \\
LDPE/PEIP & 70 & 5 & & \\
\hline
\end{tabular}




\subsection{Characterization}

Infrared spectra (IR) were recorded on a Nicolet Nexus-470 FTIR spectrometer as KBr disks. ${ }^{13} \mathrm{C}$ solid NMR and ${ }^{31} \mathrm{P}$ solid NMR spectra were recorded on a DSX 300 spectrometer (300 MHz, Bruker). TG analysis was performed on a TG 209F1 thermal analyzer at a heating rate of $20{ }^{\circ} \mathrm{C} \mathrm{min}^{-1}$ in $\mathrm{N}_{2}$ and air (Netzsch), respectively, from room temperature to $650{ }^{\circ} \mathrm{C}$. Limiting oxygen index (LOI) values were determined using an HC-2 Oxygen Index instrument on sample strips of dimensions of $120 \times 6 \times$ $3 \mathrm{~mm}^{3}$ according to ASTM D2863 (Jiang Ning Analytical Instrument Co Ltd). The combustion parameters were measured according to ISO 5660 using a cone calorimeter (Govmark) at a heat flux of $50 \mathrm{~kW} \cdot \mathrm{m}^{-2}$. The dimensions of all samples for the cone calorimeter measurements are $100 \times 100 \times 3 \mathrm{~mm}^{3}$. Morphology of the residual chars collected after the cone calorimeter tests was obtained using a S3400N field emission scanning electron microscope (Hitachi). The phase and crystallographic structures of the samples were examined by XRD on a Rigaku D/MAX 2550/PC $\mathrm{X}$-ray diffractometer working with $\mathrm{CuK} \alpha$ radiation.

\section{Results and discussion}

\subsection{Characterization of Zn-PEIP and PEIP}

Since the only difference between the Zn-PEIP samples was the variable amount of $\mathrm{Zn}^{2+}$ in the molecular structure, the structure and thermal properties of $\mathrm{Zn}$-PEIP with the highest zinc loading (Zn-PEIP-0.1) were assumed to be representative of all Zn-PEIPs. Fig. 3 shows the FTIR spectra of PEIP and Zn-PEIP-0.1. PEIP shows peaks around $1167 \mathrm{~cm}^{-1}$, attributed to uncoordinated $\mathrm{P}=\mathrm{O}$ groups, which are more abundant than in Zn-PEIP-0.1. This is due to the reduction in bond strength of the $\mathrm{P}=\mathrm{O}$ groups coordinated to $\mathrm{Zn}^{2+}$ in Zn-PEIP-0.1, shifting absorption below $1167 \mathrm{~cm}^{-1}$. At the same time, the absorption of P-O group at $1066 \mathrm{~cm}^{-1}$ does not appear to change, which indicates that coordination does not occur between $\mathrm{P}-\mathrm{OH}$ and $\mathrm{Zn}^{2+}$, only between $\mathrm{P}=\mathrm{O}$ and $\mathrm{Zn}^{2+}$. This statement is underpinned by the change of absorption 
peak of $\mathrm{NH}_{2}{ }^{+}$. Because of the presence of $\mathrm{PO}_{3} \mathrm{H}$ groups in PEIP, nitrogen atoms in PEIP can be protonated by $\mathrm{P}-\mathrm{OH}$ groups ${ }^{20}$, forming $\mathrm{NH}_{2}{ }^{+}$groups. These are expected to give two bands around 3000 and $2700 \mathrm{~cm}^{-1}$, which are usually broad, unresolved and extended to around $2200 \mathrm{~cm}^{-1} \cdot{ }^{20}$ Indeed, the IR spectrum of PEIP in Fig. 3 shows a broad and shallow band at 2000-2500 $\mathrm{cm}^{-1}$, revealing the presence of the protonated amine in PEIP. After chelation, the $\mathrm{NH}_{2}{ }^{+}$bands become weaker. Moreover, the absorption peak at $1467 \mathrm{~cm}^{-1}$, ascribed to bending of the $\mathrm{NH}_{2}^{+}$groups, is also weakened, resulting from the coordination bonds formed between $\mathrm{P}=\mathrm{O}, \mathrm{N}-\mathrm{H}$ and $\mathrm{Zn}^{2+}$. As Fig. 1 and Fig. 2 show, chelation between $\mathrm{P}=\mathrm{O}, \mathrm{Zn}^{2+}$ and $\mathrm{N}-\mathrm{H}$ groups can form two five-membered rings, resulting in a more stable chelate structure compared to chelates of just $\mathrm{P}-\mathrm{OH}$ groups and $\mathrm{Zn}^{2+}$.

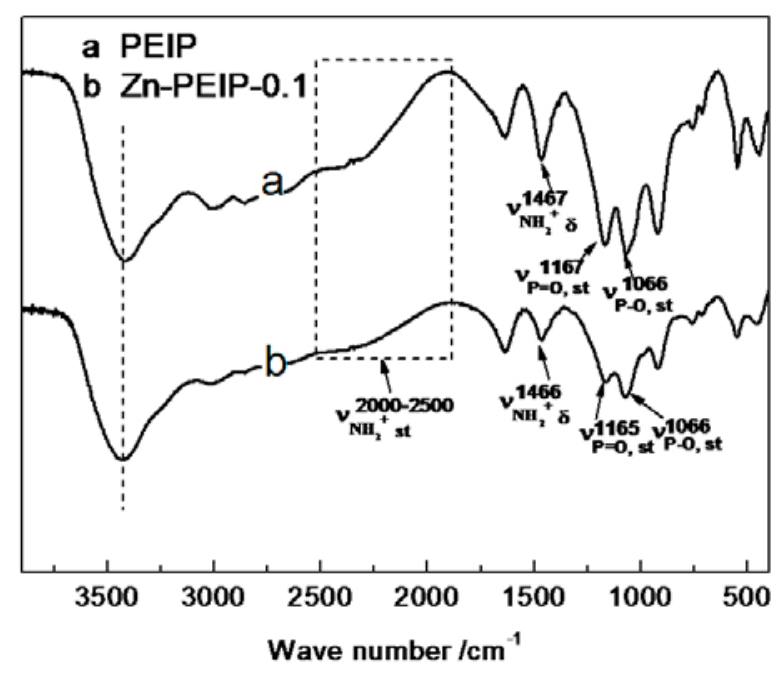

Fig. 3 IR spectra of PEIP and Zn-PEIP-0.1

The structures of PEIP and Zn-PEIP-0.1 were further characterized by solid-state ${ }^{13} \mathrm{C}-\mathrm{NMR}$ and ${ }^{31}$ P-NMR spectra as Fig. 4 (a) and (b) shows, respectively. In Fig. 4(a), there is only one obvious broad peak at $51.5 \mathrm{ppm}$ in the ${ }^{13} \mathrm{C}-\mathrm{NMR}$ spectrum of PEIP. This is because the chemical shifts of the carbons in C-C, C-P and C-N groups are similar in the PEIP, and their peaks overlap each other. After chelation, the broad peak of Zn-PEIP-0.1, shown in Fig. 4(a), shifted slightly to 51.3 ppm. This might be the result of the $\pi$-backbonding effects formed by the chelation of $\mathrm{Zn}$ and the PEIP ligand. ${ }^{31} \mathrm{P}-\mathrm{NMR}$ spectra in Fig. 4(b) also indicate the difference of the structures of PEIP and Zn-PEIP-0.1. As described earlier, both $\mathrm{NH}$ and $\mathrm{NH}_{2}{ }^{+}$groups exist in PEIP. That is to 
say, the $\mathrm{PO}_{3} \mathrm{H}$ groups may exist as it is or a $\mathrm{PO}_{3}{ }^{-}$ion, after protonation of the amine group to $\mathrm{NH}_{2}{ }^{+}$. This results in two phosphorus absorption peaks of PEIP in Fig. 4(b). Since ionization shifts $\mathrm{P}$ to a lower field, absorbance at $7.8 \mathrm{ppm}$ is attributed to $\mathrm{PO}_{3} \mathrm{H}$ and the smaller peak at $20.9 \mathrm{ppm}$ is ascribed to $\mathrm{PO}_{3}{ }^{-}$ion. After the chelation with $\mathrm{Zn}$, the ${ }^{31} \mathrm{P}$ NMR spectrum of Zn-PEIP-0.1 shows two strong absorption peaks. The unchelated $\mathrm{PO}_{3} \mathrm{H}$ peak as before shows that not all chelation sites contained zinc, while the peak at $18.3 \mathrm{ppm}$ can be assigned to $\mathrm{P}$ in the chelate of $\mathrm{P}=\mathrm{O}, \mathrm{Zn}^{2+}$ and $\mathrm{N}-\mathrm{H}$ groups, since the $\pi$-backbonding effects are formed by the chelation of $\mathrm{Zn}$ and the PEIP ligand.
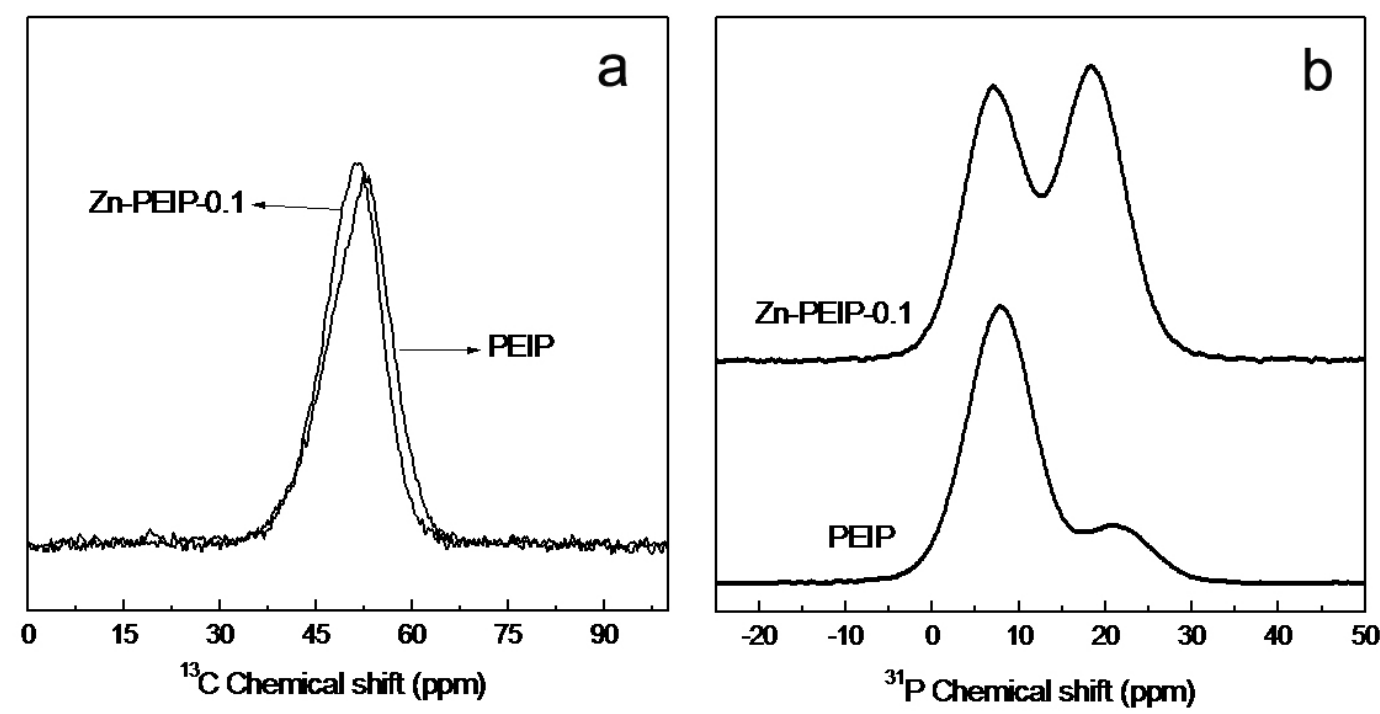

Fig. $4{ }^{13}$ C-NMR (a) and ${ }^{31}$ P-NMR (b) spectra of PEIP and Zn-PEIP-0.1

Since the zinc (II) ion may affect the thermal stability of the chelate, Zn-PEIPs with different amounts of zinc were characterized by TGA in nitrogen. Fig. 5 shows the TGA and DTG thermograms of the Zn-PEIPs. The detailed data, including the onset temperature of decomposition ( $\left.T_{\text {onset }}\right)$, maximum decomposition temperature $\left(T_{\max }\right)$ and residue percentage at $650{ }^{\circ} \mathrm{C}$, are summarized in Table 2 .

As Fig. 5 and Table 2 show, the initial decomposition temperatures ( $T_{\text {onset }}$ ) of Zn-PEIPs decrease from $228{ }^{\circ} \mathrm{C}$ to $191{ }^{\circ} \mathrm{C}$ as the amount of $\mathrm{Zn}^{2+}$ ions is reduced. As PEIP had the lowest $T_{\text {onset }}$ of $179{ }^{\circ} \mathrm{C}$, it implies that chelation between PEIP and $\mathrm{Zn}^{2+}$ increases the thermal stability of Zn-PEIPs. The residue at $650{ }^{\circ} \mathrm{C}$ follows the same trend as $T_{\text {onset }}$ of Zn-PEIPs. Zn-PEIP-0.1 has the highest amount of residue at $650{ }^{\circ} \mathrm{C}$ 
of $59.5 \mathrm{wt} \%$. If residue was composed of $\mathrm{Zn}\left(\mathrm{PO}_{3}\right)_{2}$, the residue amount would be $45 \%$, which suggests a significant proportion of the PEIP ligand contributed to the amount of residue as a char. PEIP has the smallest residue with only $26.5 \mathrm{wt} \%$, indicating the role of $\mathrm{Zn}^{2+}$ in the char forming process. According to Petersen ${ }^{21}$, divalent ions such as zinc (II) are known to promote crosslinking and dehydration reactions. During the thermal degradation of $\mathrm{Zn}-\mathrm{PEIPs}, \mathrm{Zn}^{2+}$ may catalyze the dehydration of the PEIP ligand and polyphosphoric acid will be formed. Then, $\mathrm{Zn}^{2+}$ reacts with polyphosphoric acid to form a crosslinked network through salt bridges, leading to increased residue formation. Hence, the more $\mathrm{Zn}^{2+}$ in $\mathrm{Zn}$-PEIPs, the more residue will be formed. Similar results were also found by Chen ${ }^{22}$ and Song ${ }^{23}$. They observed that nickel, zinc and chromium ions could increase the char formation in PP with an intumescent fire retardant, and they attributed this behavior to the reaction between the metal ions and the polyphosphoric acid through salt bridges.

Fig. 5b shows that Zn-PEIPs underwent multi-step thermal degradation processes. Zn-PEIP-0.1, Zn-PEIP-0.07 and Zn-PEIP-0.05 have two main weight-loss stages which can be assigned to the dehydration of PEIP and char formation. With the decrease of the chelation of $\mathrm{Zn}^{2+}$, four weight-loss stages are observed. The residue formed at about $350{ }^{\circ} \mathrm{C}$ may be unstable because there is less $\mathrm{Zn}^{2+}$, hence less crosslinking. It may continue to decompose at higher temperatures until a more stable residue forms. The less $\mathrm{Zn}^{2+}$ in $\mathrm{Zn}$-PEIPs, the closer the thermal stability of $\mathrm{Zn}$-PEIPs is to that of PEIP. Without $\mathrm{Zn}^{2+}$, dehydration of PEIP still occurs, but the degree of crosslinking may not be the same as that of the Zn-PEIPs. Therefore, the least residue is formed by PEIP. 

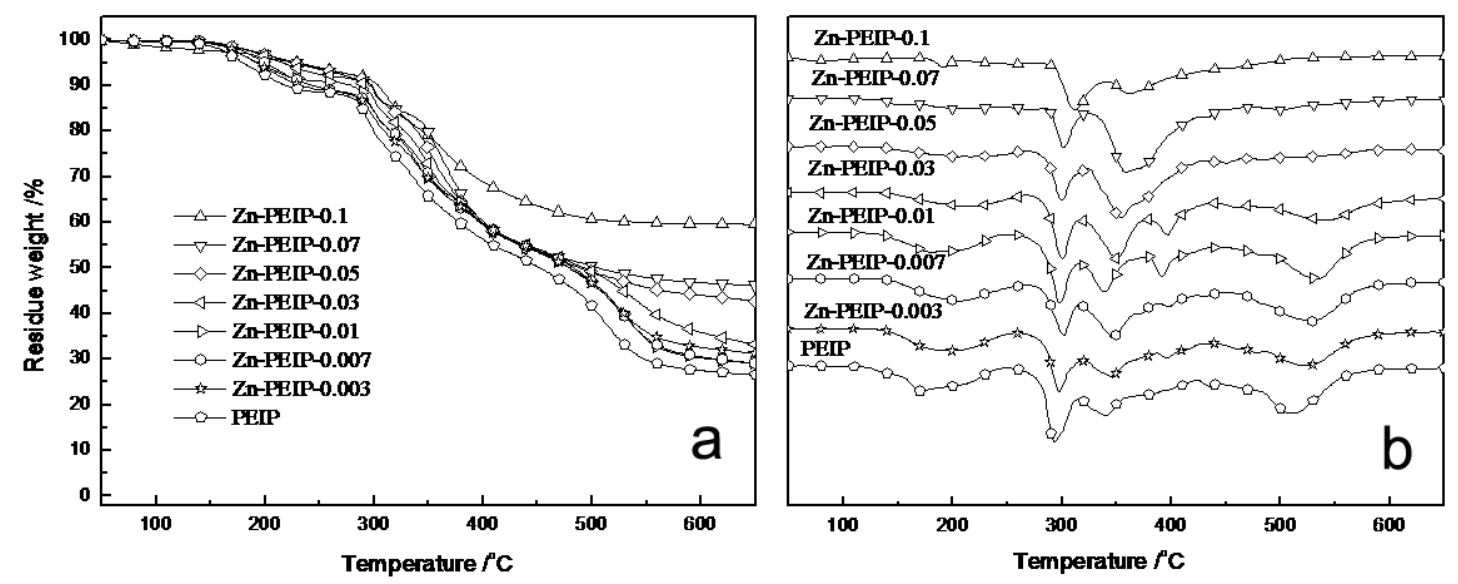

Fig. 5 TG (a) and DTG (b) curves of PEIP and Zn-PEIPs in $\mathrm{N}_{2}$

Table 2 TGA data of PEIP and Zn-PEIPs in $\mathrm{N}_{2}$

\begin{tabular}{lccc}
\hline \multicolumn{1}{c}{ Sample } & $\boldsymbol{T}_{\text {onset }} / \mathbf{}^{\mathbf{0}} \mathbf{C}^{\mathbf{a}}$ & $\boldsymbol{T}_{\max } /{ }^{\mathbf{0}} \mathbf{C}^{\mathbf{b}}$ & Residue at $\mathbf{6 5 0 ^ { \circ }} \mathbf{C} / \mathbf{w t} \mathbf{\%}$ \\
\hline Zn-PEIP-0.1 & 228 & 312,361 & 59.5 \\
Zn-PEIP-0.07 & 228 & 302,358 & 46.2 \\
Zn-PEIP-0.05 & 226 & 300,354 & 42.8 \\
Zn-PEIP-0.03 & 215 & $300,350,396,541$ & 33.2 \\
Zn-PEIP-0.01 & 193 & $298,338,391,534$ & 29.1 \\
Zn-PEIP-0.007 & 199 & $301,346,529$ & 29.1 \\
Zn-PEIP-0.003 & 191 & $297,344,520$ & 31.3 \\
PEIP & 179 & $293,340,509$ & 26.5 \\
\hline
\end{tabular}

$a$ : designated as the onset point at $5 \mathrm{wt} \%$ weight loss, b: maximum decomposition temperature

\subsection{Properties of the LDPE/Zn-PEIP and LDPE/PEIP blends}

\subsubsection{Thermal stability}

After ignition, all the oxygen at the polymer-flame interface is consumed. This means that the thermal decomposition in an inert atmosphere is the appropriate condition for determining the fuel production rate of a flaming material ${ }^{24,25}$. The thermal decomposition of LDPE and LDPE blended with PEIP and Zn-PEIPs in $\mathrm{N}_{2}$ shown in Fig. 6, best represents the fuel release. The detailed data are summarized in Table 3.

For LDPE, the degradation begins at $391{ }^{\circ} \mathrm{C}$ in $\mathrm{N}_{2}$ and the maximum weight loss rate appears at $469{ }^{\circ} \mathrm{C}$, with 0 wt \% residue at $650{ }^{\circ} \mathrm{C}$. Addition of PEIP and Zn-PEIPs results in an increase of $T_{\max }$ by $11-14{ }^{\circ} \mathrm{C}$, showing a small improvement in the thermal stability of LDPE. As described above, the crosslinks formed by Zn-PEIPs 
will lead to the formation of the protective network and enhanced charring, increasing the LDPE degradation temperature. $T_{\text {onset }}$ and the weight of residue at $650{ }^{\circ} \mathrm{C}$ decrease with the decrease of $\mathrm{Zn}^{2+}$ in $\mathrm{Zn}$-PEIPs, corresponding to that of Zn-PEIPs in $\mathrm{N}_{2}$. As expected, the lowest $T_{\text {onset }}$ of $298^{\circ} \mathrm{C}$ and the residue at $650{ }^{\circ} \mathrm{C}$ of $6.94 \mathrm{wt} \%$ is that of LDPE/PEIP without any $\mathrm{Zn}^{2+}$.
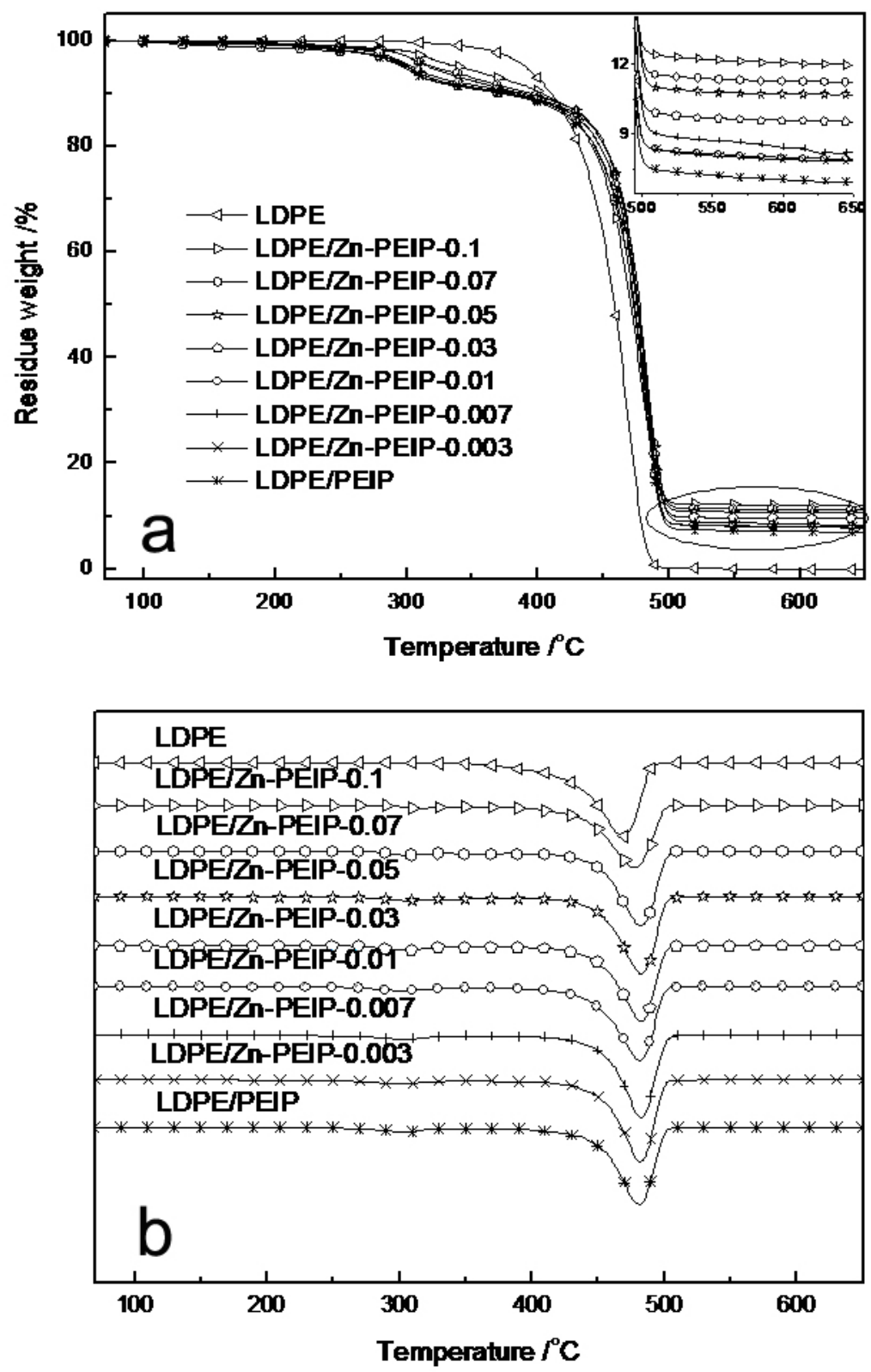

Fig. 6 TG (a) and DTG (b) curves of LDPE, LDPE/PEIP and LDPE/Zn-PEIPs in $\mathrm{N}_{2}$ 
Table 3 TGA data of LDPE, LDPE/PEIP and LDPE/Zn-PEIPs in $\mathrm{N}_{2}$

\begin{tabular}{lccc}
\hline \multicolumn{1}{c}{ Sample } & $\boldsymbol{T}_{\text {onset }} /{ }^{\circ} \mathbf{C}^{\mathbf{a}}$ & $\boldsymbol{T}_{\mathbf{m a x}} /{ }^{\mathbf{0}} \mathbf{C}^{\mathbf{b}}$ & Residue at $\mathbf{6 5 0}^{\mathbf{0}} \mathbf{C} / \mathbf{\%}^{\circ}$ \\
\hline LDPE & 391 & 469 & 0 \\
LDPE/Zn-PEIP-0.1 & 387 & 480 & 13.88 \\
LDPE/Zn-PEIP-0.07 & 330 & 483 & 11.01 \\
LDPE/Zn-PEIP-0.05 & 317 & 480 & 10.34 \\
LDPE/Zn-PEIP-0.03 & 311 & 481 & 8.28 \\
LDPE/Zn-PEIP-0.01 & 312 & 482 & 9.01 \\
LDPE/Zn-PEIP-0.007 & 308 & 482 & 8.49 \\
LDPE/Zn-PEIP-0.003 & 308 & 482 & 7.39 \\
LDPE/PEIP & 298 & 480 & 6.94 \\
\hline
\end{tabular}

a: designated as the onset point at 5 wt \% weight loss, b: maximum decomposition temperature

\subsubsection{Flammability}

Limiting oxygen index test (LOI) was conducted to investigate the flammability of the materials. It determines the minimum oxygen concentration in a mixture of oxygen and nitrogen required to support downward flame spread along the sample strip ${ }^{26}$. The results of LOI tests for LDPE and its composites are shown in Table 4. The LOI of LDPE is 17.9 indicating its inherent flammability. The addition of PEIP increased the LOI to 20.4, rising to 24.1 for LDPE/Zn-PEIP-0.01. Further increase of $\mathrm{Zn}^{2+}$ in Zn-PEIPs decreases the LOI. The increase in LOI is in contrast to the higher residue yield found by TGA.

Table 4 Results from LOI measurements

\begin{tabular}{|c|c|}
\hline Sample & LOI \\
\hline LDPE & 17.9 \\
\hline LDPE/Zn-PEIP-0.1 & 20.6 \\
\hline LDPE/Zn-PEIP-0.07 & 21.9 \\
\hline LDPE/Zn-PEIP-0.05 & 23.2 \\
\hline LDPE/Zn-PEIP-0.03 & 23.5 \\
\hline LDPE/Zn-PEIP-0.01 & 24.1 \\
\hline LDPE/Zn-PEIP-0.007 & 23.5 \\
\hline LDPE/Zn-PEIP-0.003 & 22.3 \\
\hline LDPE/PEIP & 20.4 \\
\hline
\end{tabular}

Cone calorimetry is used for investigating the burning behavior of polymeric materials, which can provide various parameters such as time to ignition $\left(t_{\text {ign }}\right)$, peak heat release rate (PHRR), total heat release (THR) and average specific extinction 
area (ASEA) ${ }^{27,28}$. Fig. 8 and Table 5 show the cone calorimetric data for LDPE, LDPE/Zn-PEIPs and LDPE/PEIP blends, respectively. These show very significant reduction in flammability for all the PEIP and Zn-PEIP materials tested.

Fig. 7 shows that LDPE burns very rapidly after ignition and a large PHRR of 1645 $\mathrm{kW} \cdot \mathrm{m}^{-2}$ appears at around 135s. In the case of LDPE/PEIP systems, PHRR and THR values are reduced by $86.3 \%$ and $30.2 \%$ with the addition of PEIP, demonstrating PEIP alone slows down the combustion process. Adding Zn-PEIPs to LDPE, the THR values decreased to an even greater extent. As shown in Table 5, the THR values for LDPE/Zn-PEIP-0.1, LDPE/Zn-PEIP-0.05 and LDPE/Zn-PEIP-0.01 are 57, 55 and 51 $\mathrm{MJ} \cdot \mathrm{m}^{-2}$, lower than that of the LDPE/PEIP blend $\left(74 \mathrm{MJ} \cdot \mathrm{m}^{-2}\right)$. The values of THR decrease with the decrease of the content of $\mathrm{Zn}^{2+}$, but increase when $\mathrm{Zn}^{2+}$ is absent from the LDPE/PEIP system. The fire behavior of LDPE in the cone calorimeter was in agreement with the result from the LOI measurement. Although LDPE/Zn-PEIP-0.01 has the highest PHRR values of the zinc containing materials, this occurred later, so had a lesser effect on the fire growth properties.

Moreover, there are notable differences in the $t_{\mathrm{ign}} \mathrm{s}$ of LDPE/Zn-PEIPs and LDPE/PEIP. The $t_{\text {ign }}$ of LDPE is $55 \mathrm{~s}$, and that for LDPE/Zn-PEIP-0.1 is prolonged to $64 \mathrm{~s}$. With the decrease of $\mathrm{Zn}^{2+}$ in LDPE/Zn-PEIPs systems, the $t_{\text {ign }}$ increase progressively. The $t_{\text {ign }}$ of LDPE/Zn-PEIP-0.01 and LDPE/PEIP are 160s and 153s, respectively, which is much longer than that of LDPE or LDPE/Zn-PEIP-0.1 or 0.05 .

According to the analysis described above, the LOI and Cone calorimetry results support each other, while TGA results of the blend are different. That is because the amounts of 5-8 mg of sample in TGA tests are so small that they cannot form an effective insulating barrier to prevent heat and fuel transfer. Although the increase of $\mathrm{Zn}^{2+}$ led to the higher residue yield found by TGA, the increased amount of char formation is not the only factor to get better flame retardancy. The swelling degree and the mechanical property of char layer are also very important factors. In order to illustrate the effect of zinc loading on flame retardant properties, a schematic char formation process is shown in Fig. 8. 

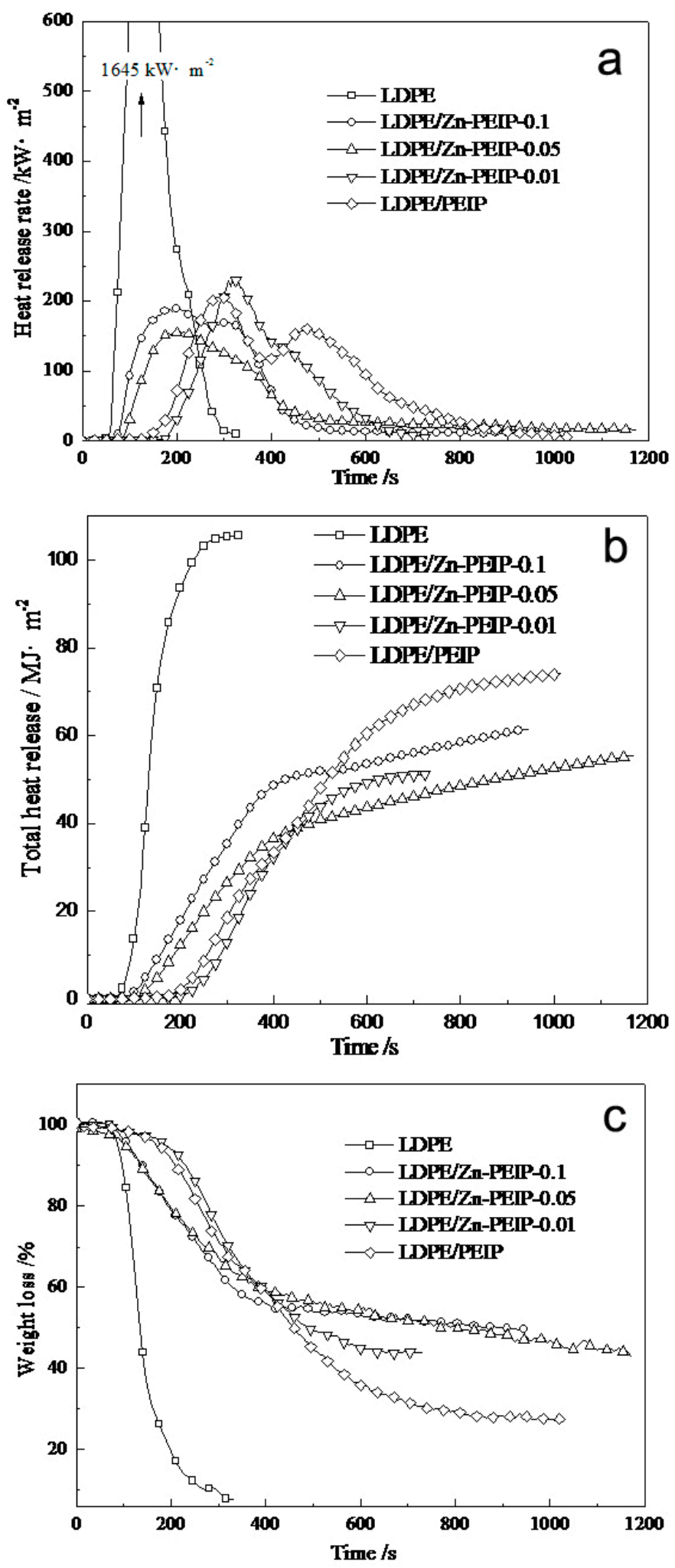

Fig. 7 (a) Heat release rate curves, (b) total heat release curves, and (c) mass loss curves of LDPE, LDPE/PEIP and LDPE/Zn-PEIPs in the cone calorimeter test 
Table 5 Combustion parameters obtained from burning in the cone calorimeter test

\begin{tabular}{lcccccc}
\hline \multicolumn{1}{c}{ Samples } & $\begin{array}{c}t_{\text {ign }} \\
\text { / }\end{array}$ & $\begin{array}{c}\text { PHRR } \\
/ \mathrm{kW} \cdot \mathrm{m}^{-2}\end{array}$ & THR $/ \mathrm{MJ} \cdot \mathrm{m}^{-2}$ & $\begin{array}{c}\text { ASEA } \\
/ \mathrm{m}^{2} \cdot \mathrm{kg}^{-1}\end{array}$ & $\begin{array}{c}\text { CO yield } \\
/ \mathrm{kg} \cdot \mathrm{kg}^{-1}\end{array}$ & $\begin{array}{c}\text { Residue } \\
/ \mathrm{wt} \%\end{array}$ \\
\hline LDPE & 55 & 1645 & 106 & 431 & 0.0557 & 6.27 \\
LDPE/Zn-PEIP-0.1 & 64 & 190 & 57 & 630 & 0.0554 & 50.37 \\
LDPE/Zn-PEIP-0.05 & 74 & 156 & 55 & 703 & 0.0673 & 43.63 \\
LDPE/Zn-PEIP-0.01 & 160 & 234 & 51 & 730 & 0.0706 & 43.97 \\
LDPE/PEIP & 153 & 226 & 74 & 877 & 0.0730 & 27.05 \\
\hline
\end{tabular}

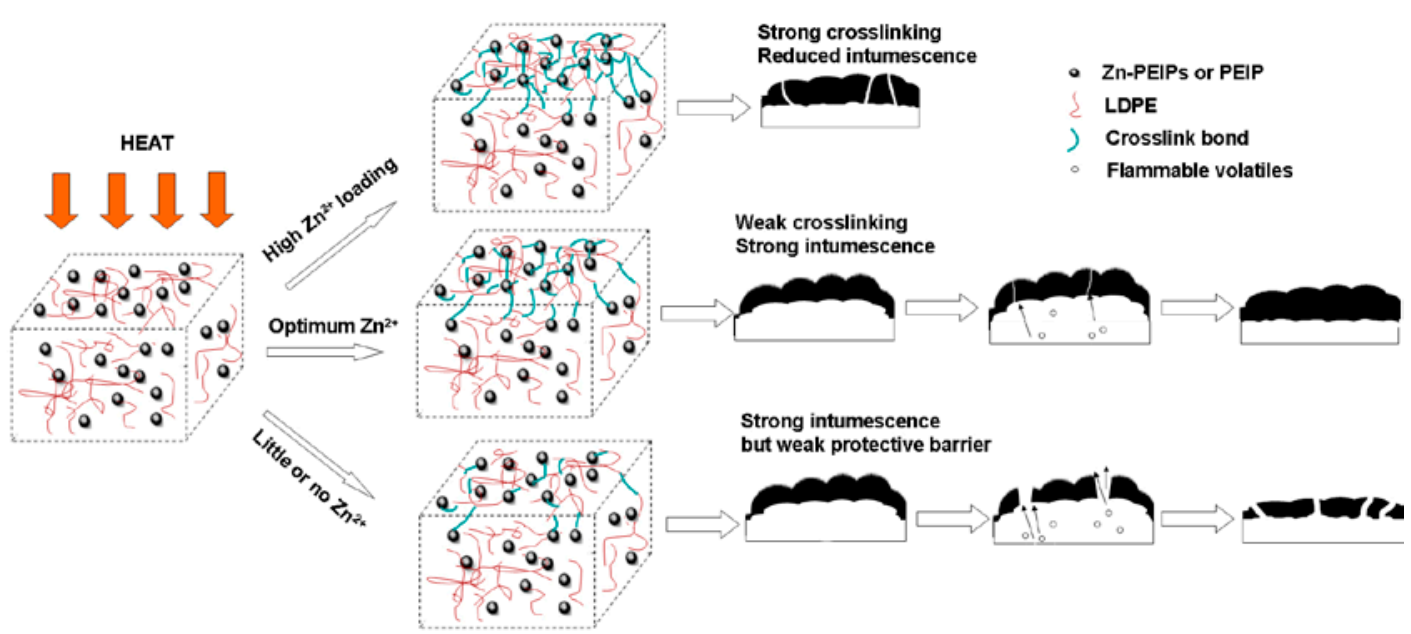

Fig. 8 Schematic of char formation process of Zn-PEIPs and PEIP in LDPE

It is generally accepted that, when heated in an atmosphere containing oxygen, LDPE chains may undergo autoxidation, with the formation of hydroperoxides and then hydroxyl groups on the main chain ${ }^{29}$. These hydroxyl groups can be phosphorylated by the degradation product of PEIP through dehydration. With the catalytic effect of $\mathrm{Zn}^{2+}$, further dehydration occurs on LDPE. The formation of salt bridges between $\mathrm{Zn}^{2+}$ and these phosphate groups may enhance crosslinking. At the same time, according to the TG analysis of Zn-PEIPs, the flame retardant Zn-PEIPs can also form a crosslinked network by the catalysis of $\mathrm{Zn}^{2+}$ during combustion. Both types of crosslinks described above are important factors to the char-formation processes, and further affect the mechanical performance of the char layer. Song found that a certain amount of metal ions and polyphosphoric acid can form a crosslinked network, through salt bridges, to form a more compact residual layer with better mechanical performance ${ }^{23}$. Thus, the char-formation processes and the quality of the protective char layer of LDPE/Zn-PEIPs blends are affected significantly by the $\mathrm{Zn}^{2+}$ loading. 
As shown in Fig. 8, when the content of $\mathrm{Zn}^{2+}$ in the fire retardant LDPE system is too high, a network with high crosslink density is formed, resulting in an increase in melt viscosity of the LDPE composite. A more rigid residue was obtained at high $\mathrm{Zn}^{2+}$ loading. As shown in Table 5, the 50.37 wt\% residue of LDPE/Zn-PEIP-0.1 is much higher than that in TGA test, demonstrating the formation of a protective barrier layer, a physical, rather than chemical fire retardant effect. PHRR and THR values of LDPE/Zn-PEIP-0.1 are also greatly decreased.

However, such a strong network may also prevent the significant swelling associated with IFRs. Due to the high melt viscosity and inflexible network, the crosslinked polymer may instead release gases through defects in its structure, such as holes or cracks, which then allow the release of flammable volatiles. The transfer of heat from the flame to the underlying substrate and the evolution of fuel from it led to the sustained combustion of LDPE. Higher THR and lower LOI values were obtained from LDPE/Zn-PEIP-0.1 than from other LDPE/Zn-PEIP systems with less $\mathrm{Zn}^{2+}$.

For the LDPE/Zn-PEIP-0.05 blend, the decrease of $\mathrm{Zn}^{2+}$ may also lower the crosslink density in the matrix. The char layer becomes more flexible and protects the underlying matrix more efficiently than that formed in the LDPE/Zn-PEIP-0.1, as shown by the higher LOI value, the prolonged $t_{\text {ign, }}$ and the lowest value of PHRR shown in Table 4 and Table 5. Due to the relatively high loading of $\mathrm{Zn}^{2+}$ in the LDPE/Zn-PEIP-0.05 blend, the THR value is not the lowest of the LDPE/Zn-PEIP systems and the flexibility of the char layer needs to be improved through further decrease of $\mathrm{Zn}^{2+}$.

With the further decrease of $\mathrm{Zn}^{2+}$ in the LDPE/Zn-PEIP-0.01, the density of network reaches an optimum value. The protective barrier layer is greatly improved with sufficient flexibility. The lowered melt viscosity of LDPE/Zn-PEIP-0.01 and the further dehydration results in a more coherent char layer being built up, which results in the highest LOI values and lowest THR value. The delay in the major peak and a slight shoulder peak on the HRR curve of LDPE/Zn-PEIP-0.01 suggest changes to the stages of char formation in the cone calorimeter.

In the LDPE/PEIP system, the LDPE chains can still be phosphorylated and form a 
network through dehydration. Without catalysis of $\mathrm{Zn}^{2+}$, the crosslink density is lower than that of LDPE/Zn-PEIPs, but the more flexible char swells better. The $t_{\text {ign }}$ of 153s of LDPE/PEIP indicates that the intumescent char is effective in protecting the polymer from being ignited. However, with the continuous attack of heat, the char layer cracks. These cracks form pathways for the escape of flammable volatiles reducing the fire retardancy. Therefore, the THR values of for LDPE/PEIP increased to $74 \mathrm{MJ} \cdot \mathrm{m}^{-2}$ compared with LDPE/Zn-PEIP-0.01, and the LOI value also decreased to the lowest value compared with the LDPE/Zn-PEIPs.

The progressive decrease of THR from LDPE/Zn-PEIP-0.1 to 0.01, while the residual mass also decreases, suggests some gas phase inhibition may also be occurring. This is supported by the significant increase in ASEA shown in Table 5. For LDPE/PEIP, the value of ASEA increases from $431 \mathrm{~m}^{2} \cdot \mathrm{kg}^{-1}$ (for LDPE) to $877 \mathrm{~m}^{2} \cdot \mathrm{kg}^{-1}$. Under the protection of the defective char layer formed by PEIP, the underlying polymer undergoes decomposition and incomplete combustion, increasing the emission of smoke. However, when the $\mathrm{Zn}^{2+}$ loading increases, the ASEA decreases gradually from $877 \mathrm{~m}^{2} \cdot \mathrm{kg}^{-1}$ to $630 \mathrm{~m}^{2} \cdot \mathrm{kg}^{-1}$, indicating that $\mathrm{Zn}^{2+}$ suppresses the production of smoke. The yield of CO also follows the same trend. Such results are consistent with our previous study 9 .

\subsubsection{Analysis of the char residue}

The optical and SEM photos of the residues after the cone calorimeter test are shown in Fig. 9 and 10, which are consistent with the hypothesis illustrated in Fig. 8. As expected, with the decrease of $\mathrm{Zn}^{2+}$ in LDPE/Zn-PEIPs system, the char layer formed in the cone calorimetric tests began to swell and become more continuous and intumescent. 

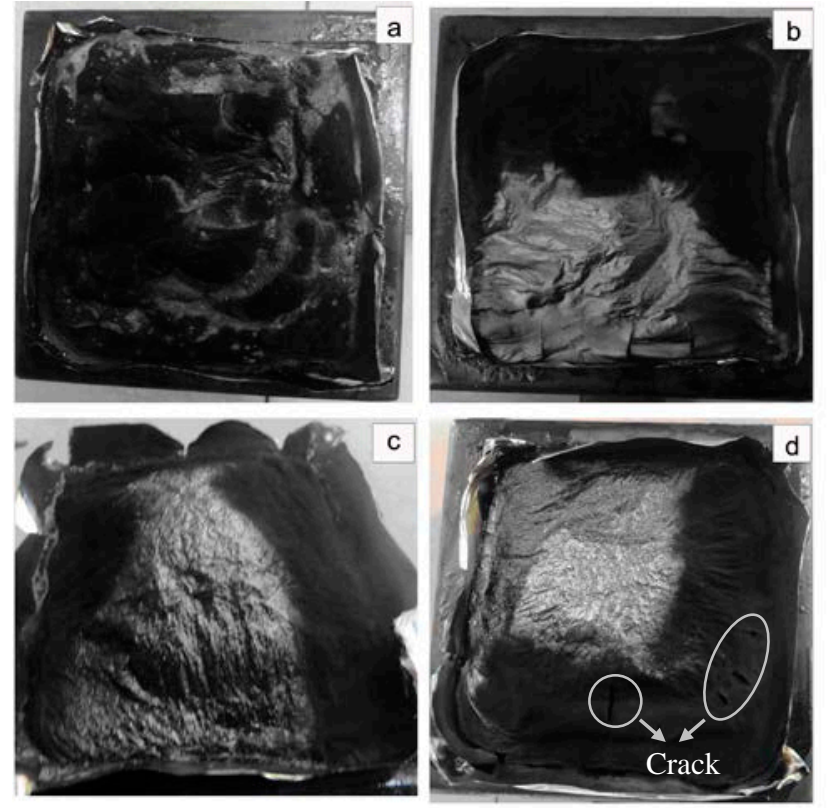

Fig. 9 Optical images of residues after the cone calorimeter test for (a)

LDPE/Zn-PEIP-0.1, (b) LDPE/Zn-PEIP-0.05, (c) LDPE/Zn-PEIP-0.01 and (d) LDPE/ PEIP 

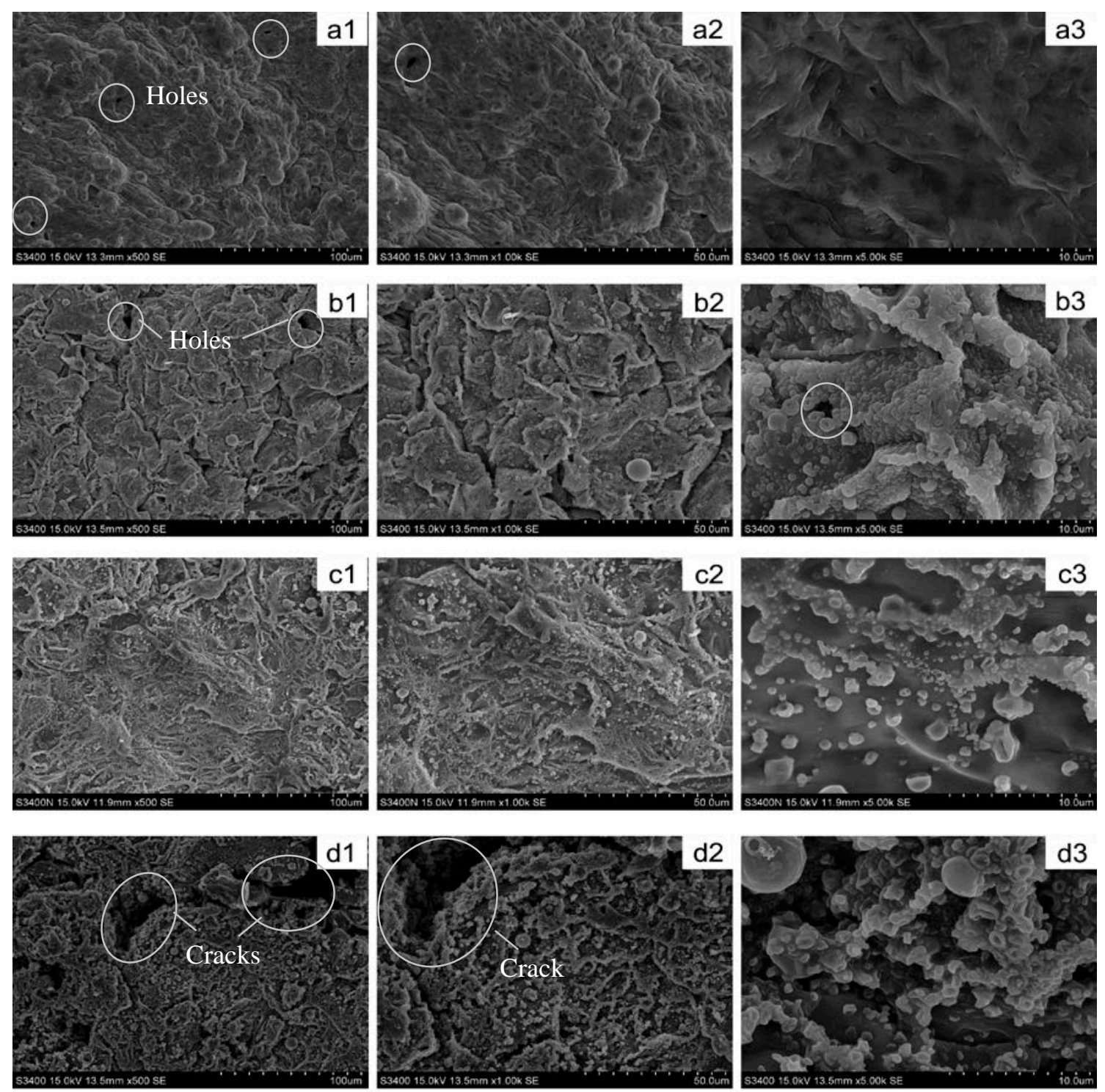

Fig. 10 SEM images of the char residues for LDPE/Zn-PEIP-0.1 (a1: $\times 500$, a2: $\times 1 \mathrm{k}$, a3 ×5k magnification); LDPE/Zn-PEIP-0.05 (b1: ×500, b2: ×1k, b3: ×5k magnification); LDPE/Zn-PEIP-0.01 (c1: ×500, c2: ×1k, c3: ×5k magnification); LDPE/PEIP (d1: ×500, d2: ×1k, d3: ×5k magnification) after the cone calorimeter test

Fig. 9(a) shows, a thick and compact char layer was formed by the LDPE/Zn-PEIP-0.1 sample. The surface morphology of the char is more like a frozen liquid with high viscosity. From the images of the surface in Fig. 10(a-1, a-2 and a-3), the chars are dense with a few holes and less obvious bubbles on the surface, because of the poor flexibility of char formed in LDPE/Zn-PEIP-0.1 system. For the LDPE/Zn-PEIP-0.5 sample, a little intumescent char layer can be seen from Fig. 9(b) 
while holes are observed in Fig. 10(b-1, b-2 and b-3).

Fig. 9 (c) shows better intumescence in the LDPE/Zn-PEIP-0.01 system. Especially in Fig. 10(c-3) at high magnification, the surface of the char layer is smooth and intact with swollen bubbles, which act as a more effective thermal barrier to reduce heat transfer from the flame to the underlying substrates, and thus reduce the total heat release rate of LDPE. Although a typical intumescent morphology is shown in Fig. 9 (d) and Fig. 10 (d-1, d-2 and d-3) for the LDPE/PEIP system, there are apparent cracks appearing on the surface of the char.

The XRD patterns of the residue of LDPE/Zn-PEIPs and LDPE/PEIP after the cone calorimeter test are given in Fig. 11. For LDPE/Zn-PEIP-0.1, the residue clearly has a significant degree of crystallinity. The peaks in the diffractogram located at $19.5^{\circ}$, $21.6^{\circ}, 23.1^{\circ}, 25.6^{\circ}, 27.1^{\circ}$ and $31.8^{\circ}$ could resulted from zinc phosphate, $\mathrm{Zn}\left(\mathrm{PO}_{3}\right)_{2}$. This suggests that $\mathrm{Zn}^{2+}$ reacts with polyphosphoric acid (decomposition products of PEIP) to form the crosslinked network. Through further dehydration, $\mathrm{Zn}\left(\mathrm{PO}_{3}\right)_{2}$ was finally formed in the residue. The XRD spectrum of LDPE/Zn-PEIP-0.05 is quite different from that of Zn-PEIP-0.1. The amorphous proportion increased, indicating a different degradation pathway of LDPE/Zn-PEIP-0.05. The peaks at $12.6^{\circ}, 21.7^{\circ}$, $28.2^{\circ}$ and $32.6^{\circ}$ are attributed to $\mathrm{ZnH}_{2} \mathrm{P}_{2} \mathrm{O}_{7}$, corresponding to degradation products with less dehydration because of the decrease of $\mathrm{Zn}^{2+}$. With further decrease of $\mathrm{Zn}^{2+}$, the XRD spectra of LDPE/Zn-PEIP-0.01 and LDPE/PEIP residue appear completely amorphous. 


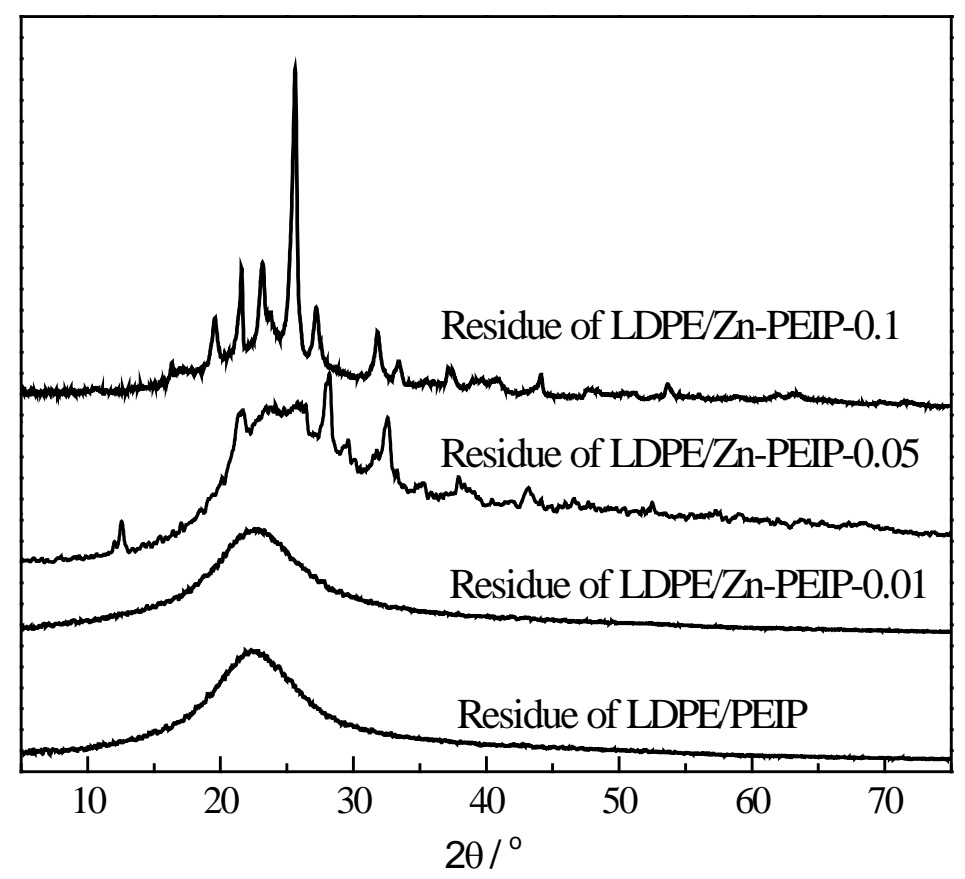

Fig. 11 XRD spectra of residues for LDPE/Zn-PEIPs and LDPE/PEIP after the cone calorimeter test

FTIR spectra of the intumescent residual for LDPE/Zn-PEIPs and LDPE/PEIP after the cone calorimeter test are shown in Fig. 12. The four residues have several peaks in common. The peak around $1630 \mathrm{~cm}^{-1}$ is assigned to stretching of phenyl, indicating the formation of polyaromatic carbonaceous species during combustion ${ }^{23}$. The broad peak at $997-1031 \mathrm{~cm}^{-1}$ is attributed to stretching of $\mathrm{P}-\mathrm{O}$ in $\mathrm{P}-\mathrm{O}-\mathrm{C}$ and $\mathrm{P}-\mathrm{O}-\mathrm{P}^{7}, 30$. The peak of LDPE/Zn-PEIP-0.1 observed at $1230 \mathrm{~cm}^{-1}$ corresponds to stretching of $\mathrm{P}=\mathrm{O}$. With the decrease of $\mathrm{Zn}^{2+}$, this becomes broader over the range $1170-1240 \mathrm{~cm}^{-1}$. The IR spectra demonstrate that Zn-PEIPs and PEIP can produce phosphoric and polyphosphoric acids during combustion, which promote dehydration forming a carbonaceous char.

The new peaks around $1400 \mathrm{~cm}^{-1}$ and the new broad peaks of $2900-3120 \mathrm{~cm}^{-1}$ in the IR spectra of LDPE/Zn-PEIP-0.05, LDPE/Zn-PEIP-0.01 and LDPE/PEIP may be attributed to the $\mathrm{C}-\mathrm{H}$ vibration of aliphatic groups ${ }^{10}$. Although more flammable, these will improve the flexibility, allowing greater intumescence and better protection with the decreasing content of $\mathrm{Zn}^{2+}$ in flame retarded LDPE. 


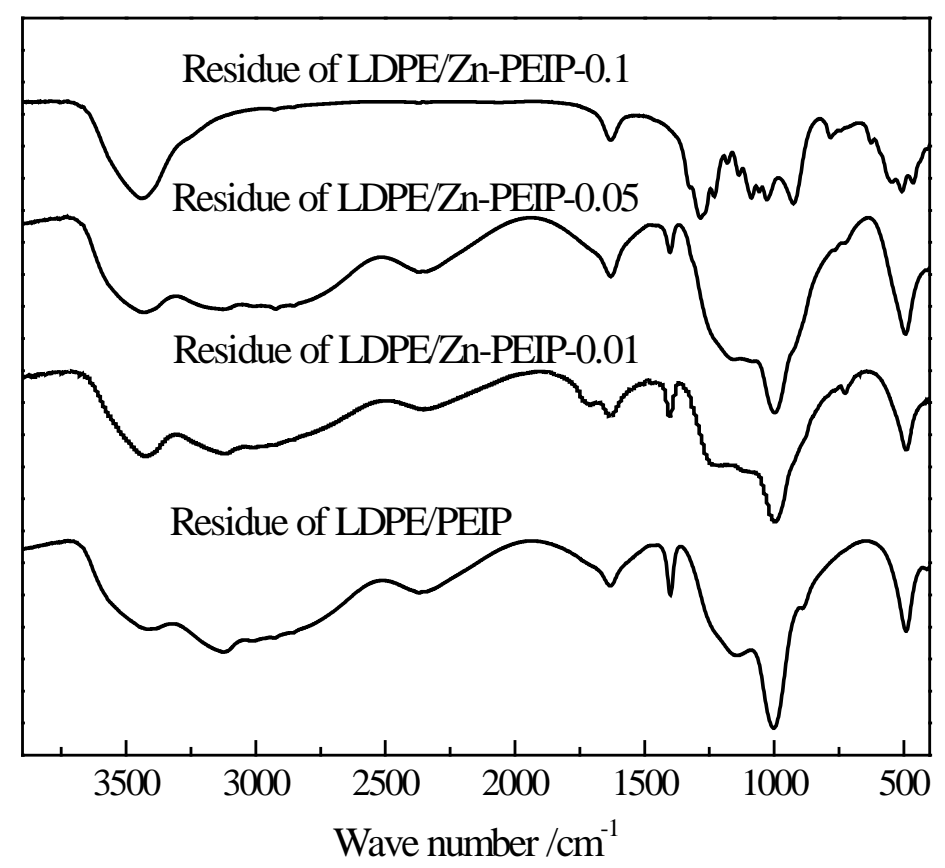

Fig. 12 IR spectra of char residues for LDPE/Zn-PEIPs and LDPE/PEIP after the cone calorimeter test

\section{Conclusions}

A novel oligomeric IFR chelate, Zn-PEIP, containing phosphorus, nitrogen and zinc was synthesized. As a consequence of the multiple coordination positions in Zn-PEIP, the content of zinc ions can be changed to influence the fire retardancy of LDPE/Zn-PEIPs. Study of thermal degradation behaviour for Zn-PEIPs and LDPE/Zn-PEIPs systems reveals that the chelation between PEIP and $\mathrm{Zn}^{2+}$ ion can improve the thermal stability of Zn-PEIPs and LDPE; the more $\mathrm{Zn}^{2+}$ in the $\mathrm{Zn}-\mathrm{PEIPs}$, the better thermal stability of Zn-PEIPs and LDPE. However, there is an optimum content of $\mathrm{Zn}^{2+}$ in LDPE/Zn-PEIP according to the LOI and cone calorimetry tests, since the presence of $\mathrm{Zn}^{2+}$ adversely affects the intumescent swelling process. More $\mathrm{Zn}^{2+}$ in Zn-PEIP may result in less swelling of the char layer. The results showed that the LDPE/Zn-PEIP-0.01 blend had the optimum content of $\mathrm{Zn}^{2+}$, which lead to the formation of the improved protective layer with flexibility and better mechanical properties, giving the lowest value of THR $\left(51 \mathrm{MJ} / \mathrm{m}^{2}\right)$ and the longest value of $t_{\text {ign }}$ (160s). Moreover, simultaneous reduction of both THR and residue yield alongside 
increase in the ASEA and the yield of CO indicate a gas phase flame retardant effect.

\section{Acknowlegements}

The authors thank Mrs. Xiurong $\mathrm{Hu}$ of Zhejiang University for the structure-matching study in XRD analysis of the char residue. The authors also greatly acknowledge the financial supports from the National Natural Science Foundation of China (No.51203136), the National Science Foundation of Zhejiang province (No. LQ12E03003) and the Scientific Special Fund of Zhejiang Province (No. 2013C01074).

\section{References}

1 Liu, Y.; Cao, Z. H.; Zhang, Y.; Fang, Z. P. Synthesis of Cerium NMorpholinomethylphosphonic Acid and Its Flame Retardant Application in High Density Polyethylene. Ind. Eng. Chem. Res. 2013, 52, 5334.

2 Bourbigot, S.; Duquesne, S. Fire retardant polymers: Recent developments and opportunities. J. Mater. Chem. 2007, 17, 2283.

3 Wang, D. Y.; Liu, Y.; Ge, X. G.; Wang, Y. Z.; Stec, A. A.; Biswas, B.; Hull, T. R.;

Price, D. Effect of metal chelates on the ignition and early flaming behaviour of intumescent fire-retarded polyethylene systems. Polym. Degrad. Stab. 2008, 93, 1024. 4 Wang, D. Y.; Liu, Y.; Wang, Y. Z.; Perdomo-Artiles, C.; Hull, T. R.; Price, D. Fire retardancy of a reactively extruded intumescent flame retardant polyethylene system enhanced by metal chelates. Polym. Degrad. Stab. 2007, 92, 1592.

5 Bourbigot, S.; Le Bras, M.; Delobel, R.; Decressain, R.; Amourex, J. P. Synergistic effect of zeolite in an intumescence process: study of the carbonaceous structures using solid-state NMR. J. Chem. Soc., Faraday Trans. 1996, 92, 149.

6 Lewin, M. Synergism and catalysis in flame retardancy of polymers. Polym. Adv. Technol. 2001, 12, 215. 
7 Braun, U.; Bahr, H.; Sturm, H.; Schartel, B. Flame retardancy mechanisms of metal phosphinates and metal phosphinates in combination with melamine cyanurate in glass-fiber reinforced poly(1,4-butylene terephthalate): the influence of metal cation Polym. Adv. Technol. 2008, 19, 680.

8 Chen, S. J.; Li, J.; Zhu, Y. K.; Guo, Z. B.; Su, S. P. Increasing the efficiency of intumescent flame retardant polypropylene catalyzed by polyoxometalate based ionic liquid. J. Mater. Chem. A 2013, 1, 15242.

9 Cao, Z. H.; Zhang, Y.; Song, P. A.; Cai, Y. Z.; Guo, Q.; Fang, Z. P.; Peng, M. A novel zinc chelate complex containing both phosphorus and nitrogen for improving the flame retardancy of low density polyethylene. J. Anal. Appl. Pyrol. 2011, 92, 339. 10 Li, N.; Xia, Y.; Mao, Z. W.; Wang, L.; Guan, Y.; Zheng, A. Influence of antimony oxide on flammability of polypropylene/intumescent flame retardant system. Polym. Degrad. Stab. 2012, 97, 1737.

11 Wehner, W. WO 2014060004 A1. April 24, 2014.

12 Wen, X.; Gong, J.; Yu, H. O.; Liu, Z.; Wan, D.; Liu, J.; Jiang, Z. W.; Tang, T. Catalyzing carbonization of poly(L-lactide) by nanosized carbon black combined with $\mathrm{Ni}_{2} \mathrm{O}_{3}$ for improving flame retardancy. J. Mater. Chem. 2012, 22, 19974.

13 Hirschler, M. M. Thermal analysis and flammability of polymers Effect of halogen-metal additive systems. Eur. Polym. J. 1983, 19, 121.

14 Cullis, C. F.; Hirschler, M. M. Char formation from polyolefins. Correlations with low-temperature oxygen uptake and with flammability in the presence of metal halogen systems. Eur. Polym. J. 1984, 20, 53.

15 Ran, S. Y.; Guo, Z. H.; Chen, C.; Zhao, L. P.; Fang, Z. P. Carbon nanotubes bridged cerium phenylphosphonate hybrids, fabrication and their effects on the thermal stability and flame retardancy of HDPE/BFR composite. J. Mater. Chem. A 2014, 2, 2999-3007.

$16 \mathrm{Li}$, J. M.; Wilkie, C. A. Improving the thermal stability of polystyrene by Friedel-Crafts chemistry. Polym. Degrad. Stab. 1997, 57, 293.

$17 \mathrm{Wu}$, N.; Ding, C.; Yang, R. J. Effects of zinc and nickel salts in intumescent flame-retardant polypropylene. Polym. Degrad. Stab. 2010, 95, 2589. 
18 Lewin, M.; Endo, M. Catalysis of intumescent flame retardancy of polypropylene by metallic compounds. Polym. Adv. Technol. 2003,14, 3.

19 Jang, J. W.; Kim, J. H.; Bae, J. Y. Effects of Lewis acid-type transition metal chloride additives on the thermal degradation of ABS. Polym. Degrad. Stab. 2005, 88, 324.

20 Bestaoui, N.; bakhmutova-Albert, E. V.; Rodriguez, A. V.; Llavona, R.;

Clearfield, A. Ab-initio Powder Structure Determination of

Dichloro[1,2-ethanediylbis(iminomethylene)bis(phosphonato)]trizinc Dihydrate. Eur.

J. Inorg. Chem. 2005, 2005, 829.

21 Petersen, H. A. in Handbook of Fiber Science and Technology. ed. M. Lewin and S.

B. Sello, Dekker: New York, 1983.

22 Chen, X. C.; Ding, Y. P.; Tang, T. Synergistic effect of nickel formate on the thermal and flame-retardant properties of polypropylene. Polym. Int. 2005, 54, 904.

23 Song, P. A.; Fang, Z. P.; Tong, L. F.; Jin, Y. M.; Lu, F. Z. Effects of metal chelates on a novel oligomeric intumescent flame retardant system for polypropylene. J. Anal. Appl. Pyrolysis. 2008, 82, 286.

24 Zhang, Y.; Chen, X. L.; Fang, Z. P. Synergistic effects of expandable graphite and ammonium polyphosphate with a new carbon source derived from biomass in flame retardant ABS. J. Appl. Polym. Sci. 2013, 128, 2424.

25 Schartel, B.; Hull, T. R. Development of fire-retarded materials-Interpretation of cone calorimeter data. Fire. Mater. 2007, 31, 327.

26 Bee, S. T.; Hassan, A.; Ratnam, C. T.; Tee, T. T.; Sin, L. T.; Hui, D. Dispersion and roles of montmorillonite on structural, flammability, thermal and mechanical behaviours of electron beam irradiated flame retarded nanocomposite. Compos. Part B-Eng. 2014, 61, 41.

27 Gallina, G.; Bravin, E.; Badalucco, C.; Audisio, G.; Armanini, M.; Chirico, A. D.; Provasoli, F. Application of cone calorimeter for the assessment of class of flame retardants for polypropylene. Fire. Mater. 1998, 22, 15.

28 Lujan-Acosta, R.; Sánchez-Valdes, S.; Ramírez-Vargas, E.; Ramos-DeValle, L.F.; Espinoza-Martinez, A. B.; Rodriguez-Fernandez, O. S.; Lozano-Ramirez, T.; Lafleur, 
P. G. Effect of Amino alcohol functionalized polyethylene as compatibilizer for LDPE/EVA/clay/flame-retardant nanocomposites. Mater. Chem. Phys. 2014, 146, 437.

29 Gugumus, F. Physico-chemical aspects of polyethylene processing in an open mixer 3. Experimental kinetics of functional group formation. Polym. Degrad. Stab. 2000, 68, 21

30 Gallo, E.; Schartel, B.; Braun, U.; Russo, P.; Acierno, D. Fire retardant synergisms between nanometric $\mathrm{Fe}_{2} \mathrm{O}_{3}$ and aluminum phosphinate in poly(butylene terephthalate). Polym. Adv. Technol., 2011, 22, 2382. 
Table of Contents

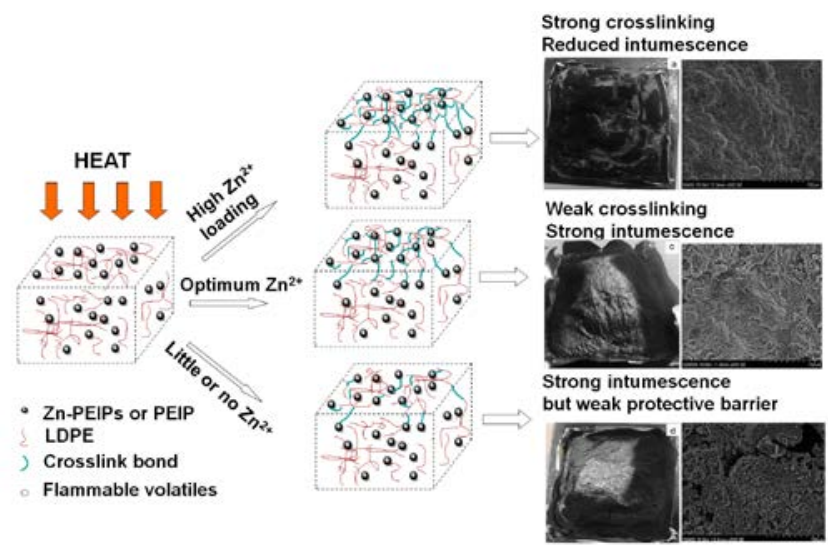

For Table of Contents Only 